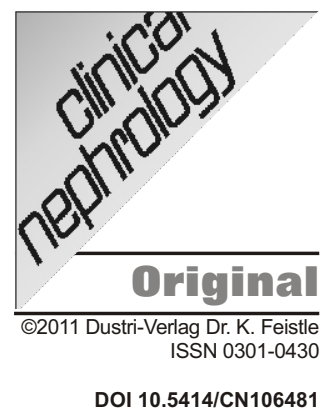

Key words

anticoagulation - atrial fibrillation - end-stage renal disease - hemorrhage - stroke
Received

September 3, 2009; accepted in revised form June 23, 2010

Correspondence to Dr. P.J. Phelan, MB BCh BAO, MRCPI

Department of Nephrology, Beaumont Hospital, Dublin 9, Ireland paulphel@gmail.com

\title{
Warfarin use in hemodialysis patients: what is the risk?
}

\author{
P.J. Phelan ${ }^{1}$, P. O'Kelly ${ }^{1}$, J. Holian ${ }^{1}$, J.J. Walshe ${ }^{1}$, C. Delany ${ }^{1}$, J. Slaby ${ }^{2}$, S. Winders ${ }^{3}$, \\ D. O'Toole ${ }^{4}$, C. Magee ${ }^{1}$ and P.J. Conlon ${ }^{1}$
}

${ }^{1}$ Department of Nephrology, ${ }^{2}$ Department of Haematology, ${ }^{3}$ Department of Computer Science, and ${ }^{4}$ HIPE Department, Beaumont Hospital, Dublin, Ireland

\begin{abstract}
Background: There is a paucity of data concerning the risks associated with warfarin in hemodialysis (HD) patients. We compared major bleeding episodes in this group with HD patients not receiving warfarin and with a cohort of non-HD patients receiving warfarin. Methods: A retrospective review of 141 HD patients on warfarin (HDW), 704 HD patients not on warfarin (HDNW) and 3,266 non-dialysis warfarin patients (NDW) was performed. Hospital admissions for hemorrhagic events and ischemic strokes were examined as was hospital length of stay and blood product use. INR variability was also assessed. Results: The incidence rates for major hemorrhage per 100 patient years was 10.8 in the HDW group as com-
\end{abstract} pared to 8.0 in the HDNW $(p=0.593)$ and 2.1 in the NDW $(p<0.001)$ groups. Mean units of red blood cell transfusions required was higher in patients on dialysis with no significant difference between HDW and HDNW groups. The risk of ischemic stroke per 100 patient years was 1.7 in the HDW group as compared to 0.7 in the HDNW groups ( $\mathrm{p}=$ $0.636)$ and 0.4 in the NDW $(p=0.003)$. The HDW group had higher inter-measurement INR variability compared to the NDW group $(p=0.034)$. In patients with atrial fibrillation, HDW group had a higher incidence of ischemic stroke than the NDW group (2.2 versus 0.4 events per 100 patient years; $p=$ 0.024). Conclusions: This study confirms the higher bleeding risk associated with HD/ESRD but suggests that warfarin use in these patients may not add significantly to this risk. We also demonstrated high rates of ischemic stroke in HD patients despite warfarin use. Summary: Our study compares the frequency of major hemorrhage and secondarily, ischemic stroke in HD patients receiving or not receiving warfarin, with non-HD patients receiving warfarin. The major finding was that frequency of hemorrhage was higher in HD patients receiving warfarin than in non-HD patients receiving warfarin, but not different in HD patients with or without warfarin. A secondary finding was that INR variability was significantly higher in HD patients than non-HD patients on warfarin.

\section{Introduction}

Uremia leads to an increased risk of bleeding due to platelet dysfunction and altered platelet aggregation. Chronic kidney disease (CKD) has been associated with a higher risk of major bleeding in patients taking warfarin [1]. Hemodialyosis (HD) patients are additionally at risk of thrombo-embolic phenomena such as vascular access thrombosis. Moreover, the prevalence of atrial fibrillation is estimated at $25 \%$ in HD patients [2]. This explains the common use of warfarin anticoagulation in the HD setting.

Warfarin is associated with appreciable levels of hemorrhagic adverse events in the general population [3] but its use in end-stage renal disease (ESRD) patients has never been fully evaluated. Holden et al. showed an increase in major bleeding episodes in HD patients who were prescribed aspirin with or without warfarin [4]. There was also more bleeding in those taking warfarin alone compared with dialysis patients taking neither aspirin nor warfarin, but this did not reach statistical significance. Recently, the influence of CKD on the hemorrhagic complications of warfarin was examined [5]. It was reported that CKD patients required a lower dose of warfarin, had instability of the INR and had a higher risk for major hemorrhage. However, only 53 of the total 565 patients had Stage IV or V CKD. We aimed to establish the risk of hemorrhage with warfarin use in our chronic HD population and to compare this group to both dialysis patients not taking warfarin and non-dialysis patients who were receiving warfarin anticoagulation. 


\section{Materials and methods}

\section{Subjects}

Our institution is the largest dialysis center in the Republic of Ireland with 220 prevalent patients. We examined prevalent, adult ( $\geq 18$ years), HD patients who received warfarin treatment for a period of at least one month, for any indication, between January 1st, 2000 and November 30th, 2008. We compared this group to all adult HD patients during this period who had never received warfarin. Only patients completing at least 90 days of HD were included in the HD groups. We also compared these patients to a cohort from the general population warfarin clinic. These were all adult patients who were maintained on warfarin for any indication for at least 1 month during the same time period. Patients were followed from initiation of warfarin until death, transplantation, transfer to $\mathrm{PD}$, transfer to another center or until the December 31st, 2008. The date of the first dialysis session was selected as the beginning of warfarin exposure when the patient was taking warfarin prior to the initiation of dialysis. HD was provided using Gambro systems (AK 200 series machines with Polyflux dialyzers). Standard anticoagulation during dialysis was delivered using unfractionated heparin, generally at a dose of $3,000-5,000$ i.u. per treatment.

\section{Data collection}

We accessed data from our Nephrology patient database (Clinical Vision 3.4a Version 1.1.34.1), the blood bank database (Mediware HCLL Version 2.9.3 accessed via Prodiver Version 5.1), the warfarin clinic database (dabl Anticoagulant) and HIPE (Hospital InPatient Enquiry) coded records. ICD-9 codes were used up to 2004 with ICD-10 codes used thereafter. The top five diagnoses were examined and these coded records were then individually adjudicated by chart review to ensure the event was a main reason for hospital admission or extended the stay if a bleed was identified that occurred during a primary admission for another cause. A major hemorrhage was defined as any bleeding episode which required hospitalization ( $\geq 1$ day) and were classed as gastro-intestinal, intra-cranial,
ENT/respiratory, or other hemorrhages. Only one hemorrhagic diagnosis was used for each inpatient stay. Ischemic stroke was defined (using ICD codes) as any non-hemorrhagic stroke which required hospital admission. The need for blood products including packed red cells and plasma were examined for the 3 groups and linked to each admission.

\section{Outcomes}

The primary outcome was defined as consisting of major bleeding episodes i.e. as any hemorrhage necessitating hospital admission. We considered events to have occurred on warfarin treatment when they occurred while the patient was receiving warfarin or within 30 days of warfarin cessation. Secondary outcome measures examined were: ischemic stroke rate, number of units of packed red cells and plasma transfused, INR variability and hospital length of stay.

\section{Statistical analysis}

Incidence rates were calculated as the number of major bleeding events divided by the total time of follow up/warfarin exposure. Poisson regression models adjusted for exposure time were used to compare incidence rates between groups. A multifactorial model included and adjusted for the confounding variables age and sex. A multiple comparison Bonferroni correction was used to allow for sub group analysis.

It is well established that warfarin has many food and drug interactions [6] which can lead to difficulty controlling prothrombin time. As HD patients who are anticoagulated have frequent measurements of the INR (at least weekly at our center), it may be expected that they experience less variation of the INR. To test for INR variability, we used a variability score proposed by Fihn et al. [7] and recently adapted by Limdi et al. [5].

$\sigma^{2}=\frac{1}{n-1} \sum_{i=1}^{n-1} \frac{(I N R i-m)^{2}}{\tau i}$

$\sigma^{2}=$ variability score, $\mathrm{n}=$ number of visits, INR $i=I N R$ at the ith visit, $m=$ mean INR for each individual patient, $\tau \mathrm{i}=$ weeks since last INR check. 
Table 1. Baseline patient characteristics with comparison of bleeding events expressed as incidence rates (events per 100 patient years).

\begin{tabular}{|c|c|c|c|c|}
\hline Group & 1. HDW & 2. HDNW & 3. NDW & $p$ value \\
\hline Patient numbers & 141 & 704 & 3266 & \\
\hline Mean age (SD) & 69.9 & 66.1 & 58.5 & 0.0001 \\
\hline \multicolumn{5}{|l|}{ Sex } \\
\hline Male (\%) & 52.5 & 63.8 & 58.2 & 0.006 \\
\hline Follow up (mean years) & 2.02 & 1.91 & 3.06 & \\
\hline \multicolumn{5}{|l|}{ Indication for warfarin } \\
\hline Atrial fibrillation & $50.0 \%$ & $\mathrm{~N} / \mathrm{A}$ & $61.0 \%$ & \\
\hline VTE & $32.0 \%$ & $\mathrm{~N} / \mathrm{A}$ & $25.0 \%$ & \\
\hline Valve replacement & $7.0 \%$ & $\mathrm{~N} / \mathrm{A}$ & $5.3 \%$ & \\
\hline Access & $6.3 \%$ & $\mathrm{~N} / \mathrm{A}$ & $\mathrm{N} / \mathrm{A}$ & \\
\hline Other & $4.7 \%$ & $\mathrm{~N} / \mathrm{A}$ & $8.7 \%$ & \\
\hline All bleeds & 10.8 & 8.0 & 2.1 & $0.593^{*}$ \\
\hline $\mathrm{GI}$ & 2.44 & 2.98 & 0.46 & $0.655^{\star}$ \\
\hline CNS & 0.00 & 0.22 & 0.21 & $0.560^{*}$ \\
\hline ENT/RESP & 2.44 & 1.93 & 0.83 & $0.573^{*}$ \\
\hline Other bleeds & 5.92 & 2.90 & 0.59 & $0.053^{*}$ \\
\hline Ischemic CVA & 1.7 & 0.7 & 0.4 & $0.636^{*}$ \\
\hline LOS (days) & 15.0 & 19.9 & 15.6 & $0.401^{*}$ \\
\hline \multicolumn{5}{|l|}{ Blood products } \\
\hline \multicolumn{5}{|l|}{ Red cells } \\
\hline$\%$ Patients transfused & 51.6 & 49.1 & 23.4 & $<0.0001^{\#}$ \\
\hline Mean units/patient & 1.23 & 1.76 & 0.5 & $0.0001^{\#}$ \\
\hline \multicolumn{5}{|l|}{ Plasma } \\
\hline$\%$ Patients transfused & 25.8 & 9.3 & 14.4 & $0.059^{\#}$ \\
\hline Mean units/patient & 0.29 & 0.29 & 0.15 & $0.350^{\#}$ \\
\hline
\end{tabular}

$\mathrm{NDW}=$ non-dialysis warfarin; HDW = hemodialysis warfarin; HDNW = hemodialysis non-warfarin; $\mathrm{SD}=$ standard deviation; VTE = venous thromboembolism (comprising DVT and PE); GI = gastrointestinal; CNS = central nervous system; ENT/RESP = ear nose throat/respiratory; CVA = cerebrovascular accident; LOS = hospital length of stay for major hemorrhage admissions. * $p$ value between Groups 1 and 2, ${ }^{*} \mathrm{p}$ value between Groups 1 and 3 .

\section{Results}

The three groups used for analysis were HDW (dialysis warfarin; 141 patients), HDNW (dialysis non-warfarin; 704 patients) and NDW (non-dialysis warfarin; 3,266 patients). Total follow up/exposure time was 285 patient years (mean 2.02 years), 1,344 patient years (mean 1.91 years) and 10,007 patient years (mean 3.06 years) respectively. Baseline demographic characteristics are shown in Table 1 . The mean age was significantly older in the two groups of patients receiving warfarin compared to the HDNW group ( $\mathrm{p}=0.0001)$. Also, the HDW group had proportionally more females with $52 / 48 \%$ male/female ratio. The HDNW group had a $64 / 36 \%$ male/female split which is consistent with the wider ESRD population in Ireland [Renal Disease in Ireland - A Strategic Review (unpublished)]. The commonest indications for warfarin were atrial fibrillation, followed by venous thromboembolism (including deep venous thrombosis and pulmonary embolism) in both groups receiving warfarin. Only $6.3 \%$ of the HDW group were prescribed warfarin for prevention of access thrombosis (Table 1). The mean INR during the exposure time was 2.05 (SD $0.44)$ in the HDW group compared to 2.54 (SD 0.83) in the NDW group $(\mathrm{p}<0.0001)$.

\section{Major bleeding complications}

For the HDW group, there were a total of 31 major bleeding episodes giving an incidence rate of 10.8 major bleeds per 100 patient years. By comparison, the HDNW group experienced 108 bleeds ( 8.0 per 100 patient years; $p=0.593$ ) and the NDW group had 209 major bleeds (2.1 per 100 patient years; $\mathrm{p}<$ $0.001)$. The difference in rates of bleeding between NDW and HDNW groups was also statistically significant $(\mathrm{p}<0.001)$.

Figure 1 shows the incidence rates of the different types of hemorrhagic complications. Intracranial hemorrhage was uncommon and did not occur in any of the dialysis patients taking warfarin. The "Other bleeds" category accounted for a significantly larger proportion of bleeds in the dialysis groups, particularly in the HDW group ( $\mathrm{p}=0.053$ versus HDNW; $p<0.001$ versus NDW). "Other bleeds" were comprised of gynecological 


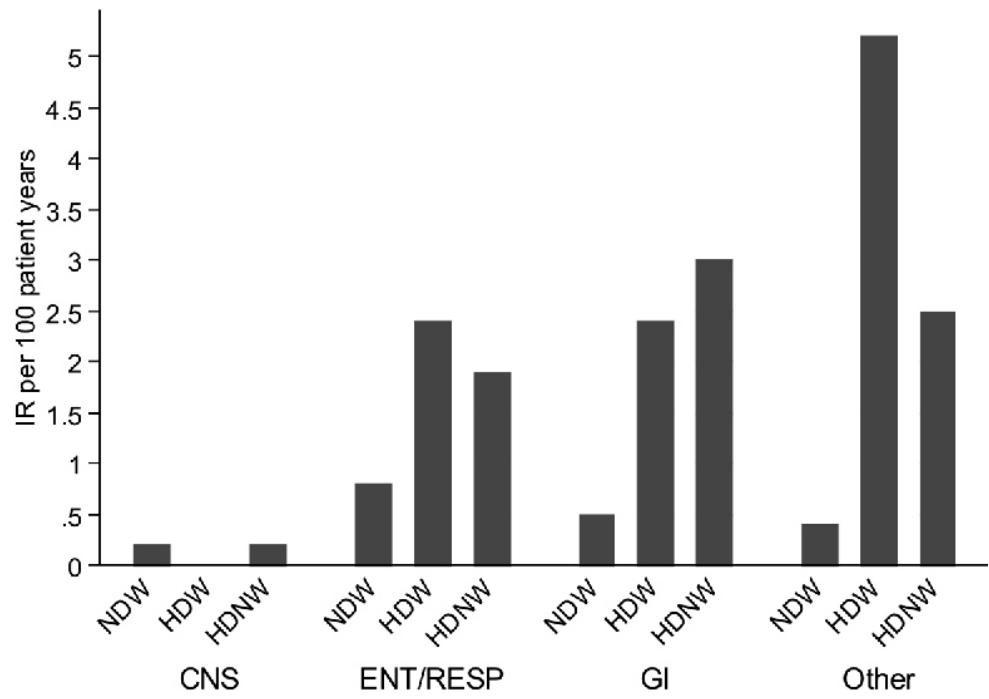

Figure 1. Bleeding incidence rates per patient group. NDW = non-dialysis warfarin; HDW = hemodialysis warfarin; HDNW = hemodialysis non-warfarin; $\mathrm{GI}=$ gastrointestinal; $\mathrm{CNS}=$ central nervous system; ENT/RESP = ear nose throat/respiratory.

Table 2. Comparison of outcomes for the three groups expressed as incidence rates (events per 100 patient years).

\begin{tabular}{|l|c|c|c|c|}
\hline Group & 1. NDW & 2. HDW & 3. HDNW & p value \\
\cline { 1 - 3 } Patient numbers & 141 & 704 & 3266 & \\
\cline { 1 - 3 } Follow-up (mean years) & 3.06 & 2.02 & 1.91 & \\
\hline All bleeds & 2.1 & 10.8 & 8.0 & 0.593 \\
\hline GI & 0.46 & 2.44 & 2.98 & 0.655 \\
\hline CNS & 0.21 & 0.00 & 0.22 & 0.560 \\
\hline ENT/RESP & 0.83 & 2.44 & 1.93 & 0.573 \\
\hline Other Bleeds & 0.59 & 5.92 & 2.90 & 0.053 \\
\hline Ischemic CVA & 0.4 & 1.7 & 0.7 & 0.636 \\
\hline LOS (days) & 15.6 & 15.0 & 19.9 & 0.401 \\
\hline
\end{tabular}

$\mathrm{NDW}=$ non-dialysis warfarin; HDW = hemodialysis warfarin; HDNW = hemodialysis non-warfarin; $\mathrm{GI}$ = gastrointestinal; $\mathrm{CNS}$ = central nervous system; ENT/RESP = ear nose throat/respiratory; CVA = cerebrovascular accident; LOS $=$ hospital length of stay for major hemorrhage admissions. ${ }^{*} p$ value between Groups 2 and 3.

Table 3. Comparison of blood products required during hospital admissions for major hemorrhage.

\begin{tabular}{|l|c|c|c|c|}
\hline Group & 1. NDW & 2. HDW & 3. HDNW & p value $^{*}$ \\
\hline Red cells \\
\hline$\%$ Patients transfused & 23.4 & 51.6 & 49.1 & $<0.0001$ \\
\hline Mean units/patient & 0.5 & 1.23 & 1.76 & 0.0001 \\
\hline Plasma \\
\hline$\%$ Patients transfused & 14.4 & 25.8 & 9.3 & 0.059 \\
\hline Mean units/patient & 0.15 & 0.29 & 0.29 & 0.350 \\
\hline
\end{tabular}

${ }^{*} p$ value between Groups 1 and 2. bleeding, major hematuria, unspecified bleeding and bleeding after a procedure.

\section{Blood product requirements}

We examined blood product requirements during each hospital admission for a hemorrhagic complication. For red cells, approximately half of both dialysis groups required blood compared to almost one quarter of the NDW group (Table 3) $(\mathrm{p}<0.001)$. Mean red cell units transfused were 1.23 units per patient in the HDW group compared to 1.76 units per patient in the HDNW group ( $\mathrm{p}=$ 0.007 ) and 0.50 units per patient in the NDW group $(\mathrm{p}<0.001)$. The proportion of patients requiring plasma transfusions were not statistically different between the groups $(\mathrm{p}=$ 0.059 between HDW and NDW). Both dialysis groups received a similar amount of plasma units which was higher than the NDW group but not significantly so $(\mathrm{p}=0.350)$.

\section{Ischemic strokes}

The overall incidence rate of ischemic strokes in all HD patients was 0.9 events per 100 patient years. There was an incidence rate of 1.7 ischemic strokes per 100 patient years in the HDW group compared to a rate of 0.4 events per 100 patient years in the NDW group (rate ratio 5.07; $\mathrm{p}=0.003$ ) (Table 2). The incidence rate in the HDNW patients was lower than that in the HDW group at 0.7 ischemic strokes per 100 patient years (rate ratio $1.80 ; \mathrm{p}=0.636$ ) but higher than the NDW group (rate ratio $1.63 ; p=0.024$ ).

When we compared only atrial fibrillation patients in the two warfarin groups, there was a higher incidence of ischemic stroke in the HD patient group as compared to the nondialysis group ( 2.2 vs. 0.4 events per 100 patient years, rate ratio $5.52 ; \mathrm{p}=0.024$ ) (Figure 2).

\section{Hospital length of stay}

Mean length of stay for hospital admissions due to bleeding was also examined. There was no statistically significant difference between the groups, with HDW having a 


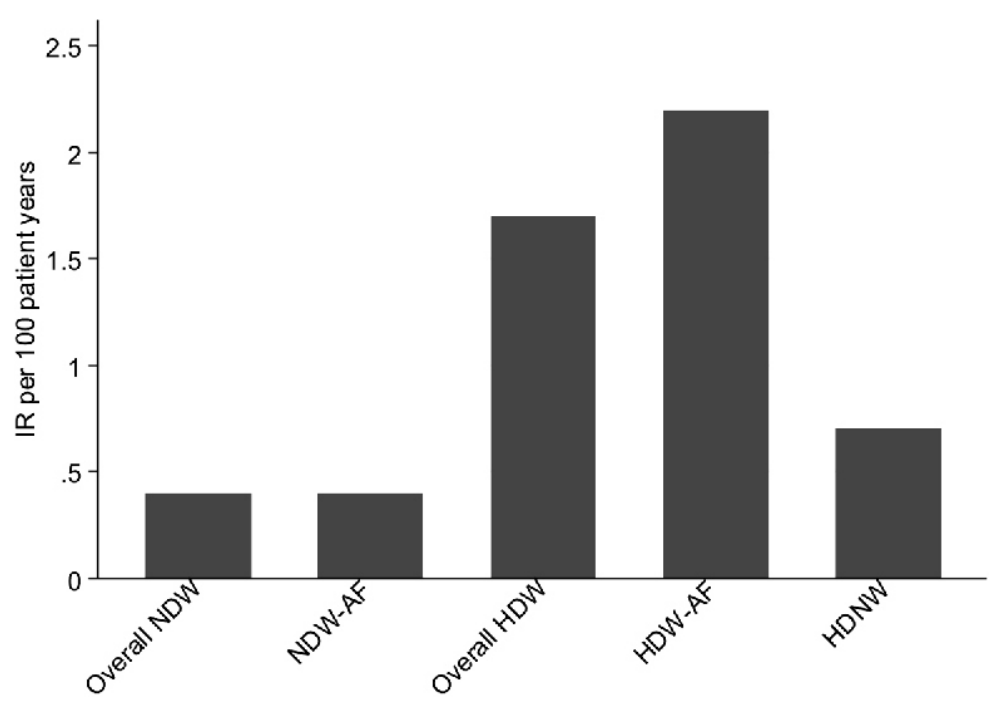

Figure 2. Incidence rates of ischemic stroke per group. NDW = non-dialysis warfarin; HDW = hemodialysis warfarin; HDNW = hemodialysis non-warfarin; $\mathrm{AF}=$ atrial fibrillation.

mean length of stay of 15.0 days compared to 19.9 days for the HDNW group and 15.6 days for NDW patients $(\mathrm{p}=0.401)$.

\section{INR variability}

The INR variability between visits was calculated as per the methods described above. HDW group had more frequent checks of their INR (mean every 13.4 days) compared to the NDW group (mean every 36.4 days). However, the mean variability was 0.25 INR units in the NDW group as compared to 0.71 INR units in the HDW group $(\mathrm{p}=0.034)$.

\section{Discussion}

There is a paucity of data concerning risks of major hemorrhage in HD patients receiving treatment with warfarin. This is demonstrated by a recent systematic review of the subject in which only five studies met initial inclusion criteria [8]. Only three small studies were randomized controlled trials and the indication for warfarin was prevention of access thrombosis. The vast majority of our patients were receiving anticoagulation for a reason other than prevention of access thrombosis. This study illustrates the high risk of major bleeding events in HD patients, irrespective of oral anticoagulation. The incidence rate of major hemorrhagic events in all HD patients at our center was 8.5 per 100 patient years. The commonest bleeding site was the GI tract, closely followed by ENT/respiratory bleeds. However, as a group, other or miscellaneous bleeds comprised the largest group in the dialysis patients treated with warfarin. Multiple bleeding sites contributed to this group including bleeding post procedure and gynecological bleeding.

Overall, the use of warfarin in our HD population appeared to be safer than we were initially expecting. There were no recorded intracranial hemorrhages in this patient group and length of hospital stay was equivalent to the other groups. Moreover, total major bleeding episodes did not occur more commonly in our HD patients receiving warfarin compared to those not anticoagulated. This perhaps should not be surprising given the results from Holden et al. showing a very low rate of intracranial hemorrhage in their HD patient group and a non-significant difference in major hemorrhages with warfarin use. In their study, it was only the aspirin groups which showed a statistically significant increase in major hemorrhagic events [4]. Also, in the study from Limdi et al. [5] looking at warfarin complications stratified by degree of CKD, patients with an eGFR $<30 \mathrm{mls} / \mathrm{min}$ per $1.73 \mathrm{~kg} / \mathrm{m}^{2}$ had significantly higher rates of major bleeding than those with better renal function. Patients with an eGFR of $30-59$ $\mathrm{mls} / \mathrm{min}$ per $1.73 \mathrm{~kg} / \mathrm{m}^{2}$ and an eGFR $>60$ $\mathrm{mls} / \mathrm{min}$ per $1.73 \mathrm{~kg} / \mathrm{m}^{2}$ had similar rates of bleeding. This suggests that factors associated with advanced CKD are responsible for the additional hemorrhagic risk [5].

The incidence of major hemorrhage in the non-dialysis patients who were treated with warfarin, at 2.3 per 100 patient years was similar to other studies in patients on warfarin for atrial fibrillation $[9,10,11]$. In these studies, a major hemorrhage was defined along similar lines to our study. Both dialysis groups had a significantly higher incidence of major hemorrhage in our study. This was true of the HDW patients despite having lower mean INRs compared to the NDW patients. The finding of similar high rates of bleeding in the dialysis groups, irrespective of warfarin use, again implies a HD/ESRD effect causing the 
high bleeding rates rather than a warfarin effect. As well as higher rates of bleeding, HD patients required more red blood cell transfusions during their admissions for hemorrhage compared to non-ESRD warfarin patients. In fact, the HDNW group actually received more red cell transfusions per patients than the HDW group. This may indicate more severe bleeding in HD patients or may reflect other issues such as a high physician tendency to transfuse HD patients who are generally sicker that the general population and with established renal anemia.

Using USRDS data [12], patients with ESRD have been shown to have a significantly higher risk of ischemic and hemorrhagic stroke compared to the general population. The incidence of ischemic strokes observed in our study was consistent with previous studies in the general HD population. These studies reported an incidence of ischemic stroke between 0.37 and 2.52 episodes/100 patient years [13, 14, 15], compared with an incidence of 0.9 events $/ 100 \mathrm{pa}-$ tient-years in our study. When we focus on the incidence of stroke in patients with atrial fibrillation, we demonstrated that the HD patients had more than 5 times the incidence rate of ischemic stroke compared to the general population with atrial fibrillation on warfarin. This may raise questions regarding the benefit, or lack of benefit of warfarin use in this group. However, to answer that question we would need to compare stroke in a HD population with atrial fibrillation who were not anti-coagulated. The evidence regarding warfarin use in ESRD patients with atrial fibrillation is contradictory. Results from the USRDS DMMS Wave II data indicated there was an improvement in all-cause mortality in patients hospitalized for atrial fibrillation who were receiving warfarin [16]. However, Wiesholzer et al. demonstrated no additional risk of stroke with atrial fibrillation in a HD patient cohort and a higher rate of stroke in those treated with warfarin [17]. Furthermore, a recent study in 1,671 incident HD patients with pre-existing atrial fibrillation reported an increased risk for new stroke associated with warfarin use [18]. This risk was not associated with taking anti-platelet agents. The results from our study certainly suggest a high risk of ischemic stroke in HD patients with atrial fibrillation.
The finding that dialysis patients have more variability in their warfarin control may initially seem surprising given that this patient group generally has more frequent INR measurements performed. However, it has been demonstrated previously that patients across a range of CKD stages require lower doses of warfarin and have poorer control of anticoagulation compared with those with an eGFR $>60 \mathrm{mls} / \mathrm{min}$ per $1.73 \mathrm{~kg} / \mathrm{m}^{2}$ [5]. They were also at a higher risk for over anticoagulation (INR $>4$ ). The probability of poor warfarin control increased with increasing severity of CKD. The explanation for increased INR variation in ESRD patients is not immediately obvious. It may be related to Vitamin $\mathrm{K}$ deficiency which has been described in HD patients [19]. Another possible reason for this finding in our study is that a different clinician often prescribes warfarin after each INR measurement for HD patients. This is in contrast to a central anticoagulation clinic which manages the general population warfarin control using a computer program to assist dosing which would likely be beneficial in the HD population. Although not demonstrated in this study, it is obvious that increased INR variability adds to the risk of hemorrhage as well as to the potential harm from under-anticoagulation.

Our results will add to the controversy surrounding warfarin use in the HD setting $[20,21,22]$. While we agree that there is no proven benefit in systemic anticoagulation for prevention of access thrombosis [23, 24], warfarin may still have a place for other, more traditional indications. Almost $94 \%$ of our HD patients received warfarin for reasons other than access-related indications. Bleeding complications, while high, were similar to other HD patients not receiving warfarin. While our study does not demonstrate additional risk of bleeding in HD patients receiving warfarin, perhaps a larger study would. Also, our study provides no information as to the potential benefit of warfarin therapy in this patient subset. Does warfarin use in HD patients reduce the incidence of thromboembolism sufficiently to justify any possible additional risk of harm? This question has not yet been fully answered but, as mentioned previously, some studies do suggest that warfarin in ESRD may be risky $[17,18]$. Future studies to elucidate these issues in the HD 
population should include randomized controlled studies of warfarin use in HD patients with atrial fibrillation and of INR variability and complications when using physician directed versus computer programme directed warfarin prescribing. Moreover, newer anticoagulant agents such as Dagibatran, which has been shown to be as efficacious as warfarin with a similar or better safety profile, will likely become more widespread in clinical practice. How these drugs, which do not need INR monitoring, will behave in HD/ESRD patients will also need to be investigated.

There are some limitations to this study. Firstly, we acknowledge the inherent weaknesses of any retrospective, single center study. Although all patient care, dialysis treatments and follow up were provided by a single center, it is possible, although unlikely, that a major bleeding episode may have occurred outside our catchment area with the patient being treated in another center. Moreover, we only included major bleeding episodes in this analysis. Therefore, minor events which did not require hospitalization, such as bleeding post removal of fistula needles, were not included. Finally, the use of anti-platelet agents was not reliably recorded in the databases, so it was not included in the study. Variations in anti-platelet use could potentially account for differences in bleeding complications between the groups.

In conclusion, we have confirmed the higher bleeding risk associated with HD compared to non-ESRD patients receiving warfarin. Moreover, we demonstrated that HD patients on warfarin had similar rates of major hemorrhage to HD patients not receiving warfarin. The need for blood products during hospital admissions for hemorrhage and length of hospital stay was also similar in these groups. These results confirm that HD patients are at high risk for bleeding but suggest that warfarin use in these patients may not add significantly to this risk.

\section{Acknowledgment}

This study was accepted as a poster presentation to the ASN 2009 meeting in San Diego under the title "Risk of Bleeding with Warfarin Anticoagulation in Haemodialysis Patients" (F-PO1256).

\section{References}

[1] Beyth RJ, Quinn LM, Landefeld CS. Prospective evaluation of an index for predicting the risk of major bleeding in outpatients treated with warfarin. Am J Med. 1998; 105: 91-99.

[2] To AC, Yehia M, Collins JF. Atrial fibrillation in haemodialysis patients: do the guidelines for anticoagulation apply? Nephrology (Carlton). 2007; 12: 441-447.

[3] Palareti G, Leali N, Coccheri S, Poggi M, Manotti $C$, D'Angelo A, Pengo V, Erba N, Moia M, Ciavarella N, Devoto G, Berrettini M, Musolesi S. Bleeding complications of oral anticoagulant treatment: an inception-cohort, prospective collaborative study (ISCOAT). Italian Study on Complications of Oral Anticoagulant Therapy. Lancet. 1996; 348: 423-428.

[4] Holden RM, Harman GJ, Wang M, Holland D, Day $A G$. Major bleeding in hemodialysis patients. Clin J Am Soc Nephrol. 2008; 3: 105-110.

[5] Limdi NA, Beasley TM, Baird MF, Goldstein JA, McGwin G, Arnett DK, Acton RT, Allon M. Kidney Function Influences Warfarin Responsiveness and Hemorrhagic Complications. J Am Soc Nephrol. 2009; 20: 912-921.

[6] Holbrook AM, Pereira JA, Labiris R et al. Systematic overview of warfarin and its drug and food interactions. Arch Intern Med. 2005; 165: 10951106.

[7] Fihn SD, McDonell M, Martin D et al. Risk factors for complications of chronic anticoagulation: A multicenter study. Warfarin Optimized Outpatient Follow-up Study Group. Ann Intern Med. 1993; 118: 511-520.

[8] Elliott MJ, Zimmerman D, Holden RM. Warfarin anticoagulation in hemodialysis patients: a systematic review of bleeding rates. Am J Kidney Dis. 2007; 50: 433-440.

[9] Stroke Prevention in Atrial Fibrillation Investigators. Bleeding during antithrombotic therapy in patients with atrial fibrillation. Arch Intern Med. 1996; 156: 409-416.

[10] Douketis JD, Arneklev K, Goldhaber SZ, Spandorfer J, Halperin F, Horrow J. Comparison of bleeding in patients with nonvalvular atrial fibrillation treated with ximelagatran or warfarin: assessment of incidence, case-fatality rate, time course and sites of bleeding, and risk factors for bleeding. Arch Intern Med. 2006; 166: 853-859.

[11] Copland $M$, Walker ID, Tait RC. Oral anticoagulation and hemorrhagic complications in an elderly population with atrial fibrillation. Arch Intern Med. 2001; 161: 2125-2128.

[12] Seliger SL, Gillen DL, Longstreth WT Jr, Kestenbaum B, Stehman-Breen CO. Elevated risk of stroke among patients with end-stage renal disease. Kidney Int. 2003; 64: 603-609.

[13] Seliger SL, Gillen DL, Tirschwell D et al. Risk factors for incident stroke among patients with endstage renal disease. J Am Soc Nephrol. 2003; 14: 2623-2631.

[14] Iseki K, Fukiyama K. Clinical demographics and long-term prognosis after stroke in patients on chronic haemodialysis. The Okinawa Dialysis Study (OKIDS) group. Nephrol Dial Transplant. 2000; 15: 1808-1813. 
[15] Kawamura M, Fijimoto S, Hisanaga S, Yamamoto Y, Eto T. Incidence, outcome, and risk factors of cerebrovascular events in patients undergoing maintenance hemodialysis. Am J Kidney Dis. 1998; 31: 991-996.

[16] Abbott KC, Trespalacios FC, Taylor AJ, Agodoa $L Y$. Atrial fibrillation in chronic dialysis patients in the United States: Risk factors for hospitalization and mortality. BMC Nephrol. 2003; 4: 1-10.

[17] Wiesholzer M, Harm F, Tomasec G, Barbieri G, Putz D, Balcke P. Incidence of stroke among chronic hemodialysis patients with nonrheumatic atrial fibrillation. Am J Nephrol. 2001; 21:35-39.

[18] Chan KE, Lazarus JM, Thadhani R, Hakim RM. Warfarin use associates with increased risk for stroke in hemodialysis patients with atrial fibrillation. J Am Soc Nephrol. 2009; 20: 2223-2233.

[19] Pilkey RM, Morton AR, Boffa MB, Noordhof C, Day AG, Su Y, Miller LM, Koschinsky ML, Booth SL. Subclinical vitamin K deficiency in hemodialysis patients. Am J Kidney Dis. 2007; 49: 432-439.

[20] Vázquez E, Sánchez-Perales C, García-Cortes MJ et al. Ought dialysis patients with atrial fibrillation be treated with oral anticoagulants? Int J Cardiol. 2003; 87: 135-141.

[21] Bennett WM. Should dialysis patients ever receive warfarin and for what reasons? Clin J Am Soc Nephrol. 2006; 1: 1357-1359.

[22] Wright JR, Kalra PA. Ought dialysis patients with atrial fibrillation be treated with anticoagulants? Int J Cardiol. 2003; 87: 139-141.

[23] Crowther MA, Clase CM, Margetts PJ et al. Low-intensity warfarin is ineffective for the prevention of PTFE graft failure in patients on hemodialysis: a randomized controlled trial. J Am Soc Nephrol. 2002; 13: 2331-2337.

[24] Mokrzycki MH, Jean-Jerome K, Rush H, Zdunek $M P$, Rosenberg SO. A randomized trial of minidose warfarin for the prevention of late malfunction in tunneled, cuffed hemodialysis catheters. Kidney Int. 2001; 59: 1935-1942. 